\title{
Boerhaave syndrome: an unusual cause of bilateral exudative pleural effusion
}

\author{
Rishabh Goel', Benhur Joel Shadrach², Ritesh Kumar Nayak², Anukool Jain ${ }^{4}$ \\ 'Department of Pulmonary Medicine, Shahi Global Hospital, Gorakhpur, India \\ ${ }^{2}$ Department of Pulmonary Medicine, All India Institute of Medical Sciences, Jodhpur, India \\ ${ }^{3}$ Consultant Radiologist Purvanchal Diagnostic Centre, Gorakhpur, India \\ ${ }^{4}$ Consultant Pulmonologist, Department of Pulmonary Medicine, Pushpanjali Hospital, Agra, India
}

A 45-year-old male, security guard, chronic alcoholic, presented to us with complaints of low-grade fever, recurrent vomiting, bilateral pleuritic chest pain, dry cough, and progressive breathlessness for the past 4 days. On admission, he had tachycardia, tachypnoea, and slightly reduced peripheral oxygen saturation. Arterial blood gas revealed mild hypoxaemia and respiratory alkalosis. Chest radiograph showed bilateral mild to moderate pleural effusion (left > right). Pleural fluid analysis revealed an exudative, neutrophilic predominant effusion with low adenosine deaminase levels and sterile cultures. He developed subcutaneous emphysema during his hospital stay. Computed tomography (CT) of the thorax disclosed a transmural tear in distal thoracic oesophagus resulting in pneumomediastinum and bilateral pleural effusion (Figure 1). Contrast oesophagography identified leakage in the left lower lateral wall of the distal intrathoracic oesophagus. Additional pleural fluid cytobiochemical analysis revealed undigested food particles, low $\mathrm{PH}$ and elevated amylase levels consistent with our diagnosis. The patient underwent primary surgical oesophageal repair and bilateral tube thoracostomy.

Pleural effusion is defined as a pathological accumulation of fluid in the pleural cavity due to an imbalance between its formation and removal. Light's criteria can be used to classify them into exudative and transudative effusions. Bilateral effusions are usually transudative, the most common cause includes congestive heart failure, while malignancy is considered the most prevalent reason for bilateral exudative effusions. Other common causes of bilateral effusions include chronic liver disease, chronic kidney disease, hypalbuminaemia, parapneumonic effusions, autoimmunity, tuberculosis, and a combination of etiologies [1]. Oesophageal rupture, which can be spontaneous or secondary to trauma/iatrogenic, is an uncommon cause of bilateral exudative pleural effusions seen in less than $10 \%$ of such cases [2].

Boerhaave syndrome (spontaneous oesophageal rupture) is a medical emergency characterised by Mackler's triad of vomiting, subcutaneous emphysema and chest pain [2]. Pathophysiology involves a sudden increase in intraluminal oesophageal pressure due to vomiting/retching leading to a transmural perforation of the oesophagus, predominantly occurring along the left posterolateral wall of the distal oesophagus, 3-6 cm above the oesophageal hiatus of the diaphragm [3]. It usually occurs between 20-40 years of age with male predominance and risk factors including alcoholism, gastroesophageal reflux, peptic ulcer, hiatus hernia, and neurological disorders [4]. Chest radiograph findings include pleural effusions (usually left-sided), hydropneumothorax, pneumomediastinum, subcutaneous emphysema, and may be normal in approximately $10 \%$ of the cases. CT thorax helps demonstrate communication of the oesophagus with the mediastinum and/or the pleural cavity [5]. Diagnosis is confirmed by contrast oesophagography, depicting leakage of contrast from the oesophagus and identifying the anatomical site of perforation [2]. Management options include surgical, endoscopic, and conservative approach with surgery regarded as the first choice especially if done within 24 hours. Endoscopic line of action involves placement of self-expanding metallic stents. Finally, conservative approach requires intravenous fluids, antibiotics, tube thoracotomy, and nutritional supplementation [5]. Prognosis is poor with an overall mortality rate of $20-40 \%$ [3].

Our report highlights a rare yet life-threatening cause of a bilateral exudative pleural effusion and alerts the clinician to the importance of early diagnosis and definitive management, which is paramount in reducing morbidity and mortality.

Address for correspondence: Benhur Joel Shadrach, Department of Pulmonary Medicine, All India Institute of Medical Sciences, Jodhpur, India; e-mail: benjoe6326@gmail.com

DOI: 10.5603/ARM.a2021.0005

Received: 11.10.2020

Copyright (C) 2021 PTChP

ISSN 2451-4934

Conflict of interest: None declared 

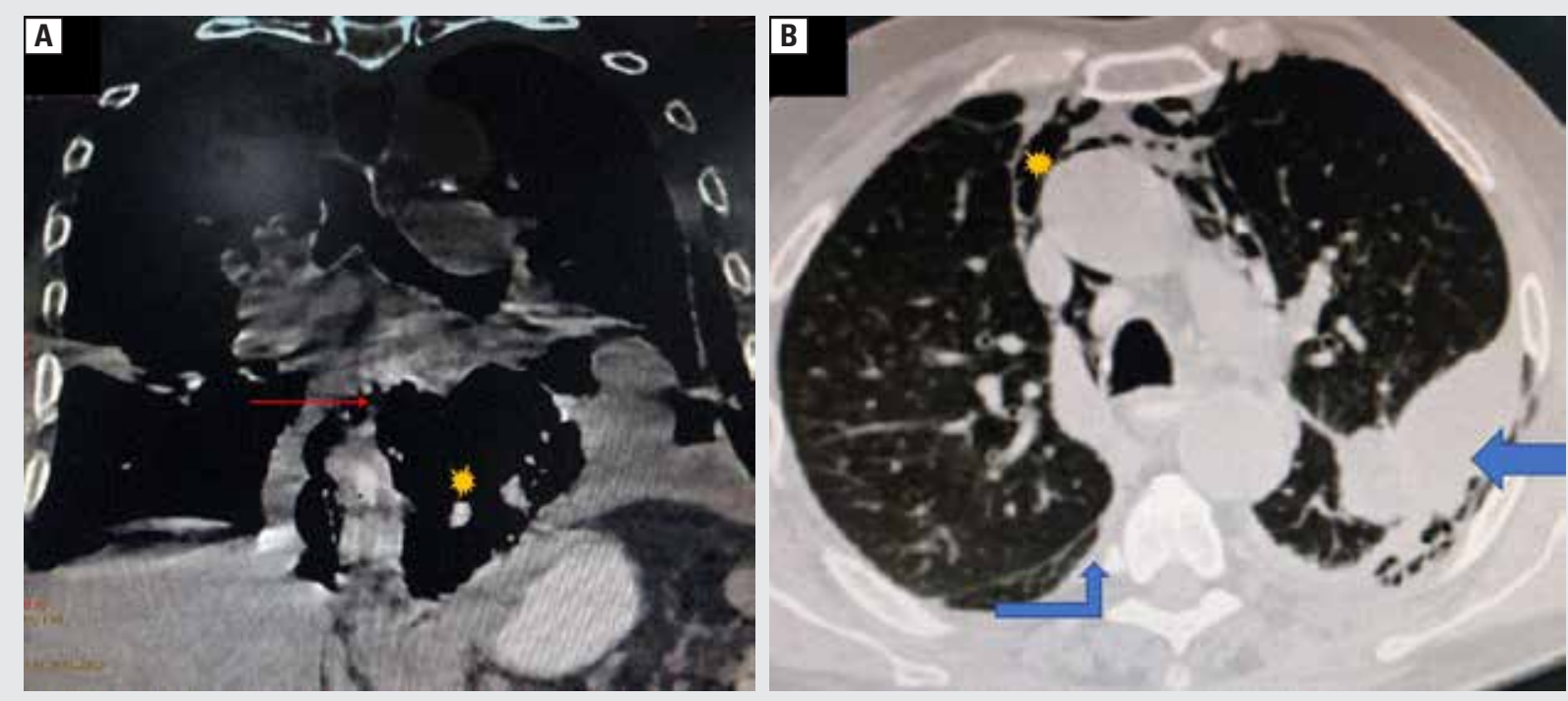

Figure 1. A. Coronal reformatted CT thorax image shows a small transmural defect in the left lateral wall of the distal thoracic oesophagus caudal to the gastroesophageal junction (red arrow) with leakage of oesophageal contents into the mediastinum and associated pneumomediastinum (yellow asterix); B. Axial reformatted CT thorax image at the level of the aortic arch shows bilateral pleural effusion (blue arrows) and pneumomediastinum (yellow asterix)

\section{References:}

1. Puchalski JT, Argento AC, Murphy TE, et al. Etiologies of bilateral pleural effusions. Respir Med. 2013; 107(2): 284-291, doi: 10.1016/i.rmed.2012.10.004, indexed in Pubmed: 23219348.

2. Rassameehiran S, Klomjit S, Nugent K. Right-sided hydropneumothorax as a presenting symptom of Boerhaave's syndrome (spontaneous esophageal rupture). Proc (Bayl Univ Med Cent). 2015; 28(3): 344-346, doi: 10.1080/08998280.2015.11929269, indexed in Pubmed: 26130884.

3. de Schipper JP, Pull ter Gunne AF, Oostvogel HJM, et al. Spontaneous rupture of the oesophagus: Boerhaave's syndrome in 2008. Literature review and treatment algorithm. Dig Surg. 2009; 26(1): 1-6, doi: 10.1159/000191283, indexed in Pubmed: 19145081.

4. Vallböhmer D, Hölscher AH, Hölscher M, et al. Options in the management of esophageal perforation: analysis over a 12-year period. Dis Esophagus. 2010; 23(3): 185-190, doi: 10.1111/j.1442-2050.2009.01017.x, indexed in Pubmed: 19863642.

5. Korczynski P, Krenke R, Fangrat A, et al. Acute respiratory failure in a patient with spontaneous esophageal rupture (Boerhaave syndrome). Respir Care. 2011; 56(3): 347-350, doi: 10.4187/respcare.00900, indexed in Pubmed: 21255506. 